Trauma Berufskrankh $2003 \cdot 5$ [Suppl 2]: S285-\$289 DOI 10.1007/s10039-003-0714-3

Unterschenkel- und obere Sprunggelenksfrakturen

Hans Zwipp · René Grass

Klinik und Poliklinik für Unfall- und Wiederherstellungschirurgie, Universitätsklinikum Carl Gustav Carus, Technische Universität Dresden, Dresden

\title{
Rekonstruktion nach Fehlheilung der Pilon-tibiale- und oberen Sprunggelenkfraktur
}

ährend einige wenige Autoren [4,5, 7] bei kleinen Fallzahlen in bis zu $85 \%$ der Fälle sehr gute und gute Resultate nach der Korrektur fehlverheilter Knöchelbrüche erzielen konnten, zeigte eine große Sammelstudie mit 135 Fällen von 6 deutschen AO-Kliniken [10] eine ernüchternde Anzahl unbefriedigender Ergebnisse: $48 \%$ nach Weber-B-, $57 \%$ nach Weber-C- und $80 \%$ nach Pilontibiale-Fraktur. Dies wird zusätzlich dadurch akzentuiert, dass ausschließlich extraartikuläre Fehlheilungen wie Pseudarthrosen $(81,5 \%)$, Fibulaverkürzungen (29,6\%), Bandinstabilitäten (23,7\%) oder supramalleoläre Fehlstellungen (11,1\%) korrigiert wurden. Während die Autoren dieser Sammelstudie eine eindeutige Abhängigkeit des operativen Ergebnisses vom Zeitintervall zwischen dem Trauma und der Korrektur, vom Alter der Patienten und vom Ausmaß der Fehlstellung sahen, konnte Rosen [7] lediglich einen direkten Zusammenhang zum Ausmaß des Korrekturgewinns und zum Zustand des Knorpels zur Zeit der Korrekturoperation sehen.

Nach unseren eigenen ermutigenden Ergebnissen muss die Wertigkeit von Korrekturoperationen am Sprunggelenk nach Fehlheilung erneut geprüft werden, da uns heute CT, MRT, Sonographie, standardisierte Belastungsaufnahmen im Stehen sowie ein neues Syndesmosenstressgerät zum einen die Pathologie der Fehlverheilung exakter zeigen und uns diese neuen Bild gebenden Verfahren, v. a. die präoperative CT-Analy- se, zum anderen eine präzise Korrekturplanung erlauben.

\section{Eigenes Krankengut}

In der Zeit von Oktober 1993-Dezember 1999 wurden an der Klinik für Unfallund Wiederherstellungschirurgie am Universitätsklinikum „Carl Gustav Carus" insgesamt 37 Patienten einer Korrekturoperation am Sprunggelenk, im Mittel 15 Monate nach dem Trauma bzw. der Erstversorgung (6 Wochen-49 Monate), zugeführt. Dabei wurden entsprechend der Lokalisation der Fehlheilung oder Nichtverheilung (Pseudarthrosen) 3 Gruppen unterschieden:

a) supramalleolar $(n=4)$,

b) malleolar inklusive Syndesmoseninsuffizienz $(n=30)$ und

c) intraartikulär $(n=3)$.

Die Korrekturoperation hat bei 29 kontrollierten Patienten (im Mittel 2,9 Jahre postoperativ) in allen Fällen zur signifi-

\footnotetext{
(c) Springer-Verlag 2003

Prof. Dr. Hans Zwipp

Klinik und Poliklinik für Unfallund Wiederherstellungschirurgie, Universitätsklinikum Carl Gustav Carus, Technische Universität Dresden, Fetscherstraße 74,01307 Dresden, Tel.: 0351-4583777, Fax: 0351-4584307, E-Mail:uwc@rcs.urz.tu-dresden.de
} 
Trauma Berufskrankh $2003 \cdot 5$ [Suppl 2]: S285-S289 DOI 10.1007/s10039-003-0714-3

\section{H. Zwipp · R. Grass}

\section{Reconstruction after malunion of tibial pilon and ankle joint fractures}

\section{Abstract}

The results achieved with reconstructive procedures in 37 patients after malunion of an ankle joint or tibial pilon fracture, performed between October 1993 and December 1999 at the Clinic for Traumatology and Reconstructive Surgery of the University Hospital "Carl Gustav Carus" in Dresden, are highly encouraging. The patients were treated 6 weeks to 49 months after the traumatic event or emergency care. This applies especially to malleolar correction including cases of chronic syndesmotic insufficiency $(n=30)$. Supramalleolar $(n=4)$ and intra-articular joint-sparing $(n=3)$ operations also appear to be worthwhile since clinical and radiological follow-up after an average of 2.9 years resulted in significant improvement of subjective complaints and objective radiological criteria. During this period, only one reoperation was necessary and no arthrodesis was observed. Preoperative CT analysis proved to be invaluable for understanding pathological features and planning a detailed corrective procedure.

\section{Keywords}

Ankle joint · Pilon · Malunion · Nonunion Late corrections

\section{Unterschenkel- und obere Sprunggelenksfrakturen}

kanten Verbesserung geführt und im genannten Beobachtungszeitraum nur eine Nachkorrektur, jedoch keine Arthrodese erfordert.

\section{Ätiologie, Pathogenese und Klassifikation}

Insgesamt werden nach konservativer oder operativer Therapie von OSG- und Pilon-tibiale-Frakturen 5 Gruppen der Fehl- und Nichtverheilung unterschieden:

1. supramalleolare Fehl- bzw. Nichtverheilung

2. mono-, bi- und trimalleolare Fehlverheilung bzw. Pseudarthrose

3. isolierte oder mit Verkürzung bzw. Fehlrotation der Fibula einhergehende distale Syndesmoseninsuffizienz

4. Verstarrung der distalen Syndesmose durch heterotope Ossifikation mit Rotationsverlust der Fibula

5. direkte Gelenkstufenbildung nach Supinations-/Adduktions-Impressions-Fraktur, Pilon-tibiale-Fraktur, Tubercule-de-Chaput- oder hinterer Volkmann-Fraktur

Die Entwicklung einer Falschgelenkbildung im Bereich des oberen Sprunggelenks nach Luxationsfraktur ist heute selten. Sie wurde in den $50 e r$ Jahren bei konservativer Behandlung von Pronationsbrüchen in bis zu 62,8\% der Fälle [6] gesehen. In den 7oer Jahren wurde nach einer österreichischen Sammelstudie [2] die Pseudarthrose des Innenknöchels unter konservativer Behandlung 5-mal häufiger beobachtet als die des Außenknöchels. Untersuchungen von Bauer et al. [1] zeigten in einer 30-Jahrebrüchen nur 2 Pseudarthrosen des Innenknöchels und keine des Außenknöchels, wodurch die allgemeine Beobachtung in der Literatur bestätigt wird, dass mit zunehmender operativer Behandlung der Sprunggelenkbrüche die Tendenz zur Pseudarthrosenentwicklung eindeutig abgenommen hat.

Pseudarthrosen nach operativer Behandlung treten - wenn überhaupt nach primär offener Fraktur mit Knochendefekt, drittgradig geschlossenen Trümmerfrakturen mit Störung der Biologie, nach fehlerhafter Osteosynthesetechnik oder im Rahmen perioperativer Infektionen auf [12].

Während die supramalleolare Pseudarthrose heutzutage kaum mehr gesehen schweren Polytraumen mit notgedrungener konservativer Behandlung oder nach Crushverletzung kindlicher Sprunggelenkfrakturen mit einseitigem vorzeitigem Fugenverschluss oder Fehlheilungen der Fibula mit Verkürzung und/oder isolierter Syndesmoseninsuffizienz nach Maisonneuve-Frakturen, Pronationsund Eversionsfrakturen auch heute noch relativ häufig beobachtet [3]. Zur Prävention einer Syndesmoseninsuffizienz wurde gefordert, dass bei notwendigem Setzen einer Syndesmosenstellschraube „de principe“ ein postoperatives CT mit koronarer Schichtung durchgeführt wird, um eine Fehlstellung der Fibula in der Incisura fibularis tibiae auszuschließen [3]. Statistik mit insgesamt 1784 Knöchelwird, werden Fehlverheilungen nach
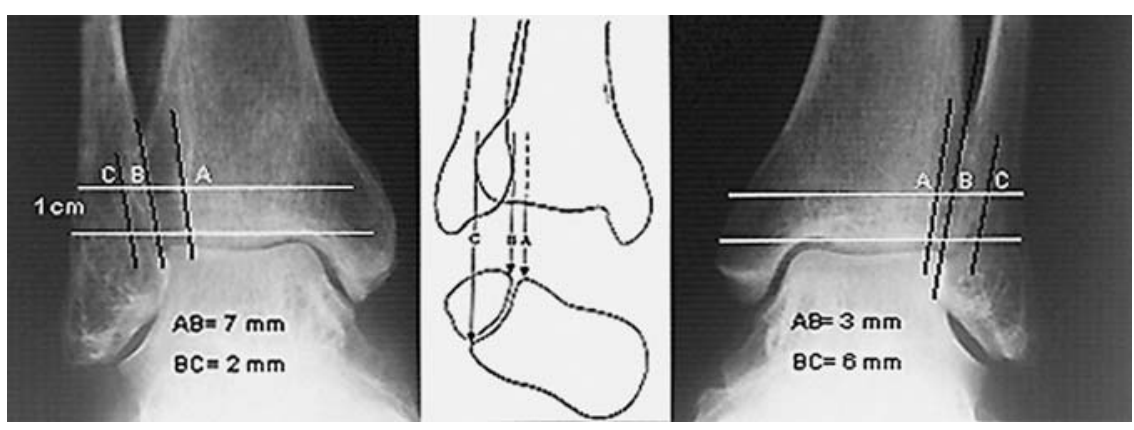

Abb. $1 \Delta$ OSG-a.-p.-Aufnahme im Stehen beidseits mit $20^{\circ}$ Innenrotation zum Nachweis einer chronischen distalen Syndesmoseninsuffizienz. Die radiologische Kontur $A$ entspricht dem Tuberculum tibiale posterius, $B$ dem medialen Rand der Fibula, $C$ der lateralen Begrenzung des Tuberculum tibiale anterius. Die Distanzen für den so genannten Espace claire und die tibio-fibulare Überlappung werden definitionsgemäß $1 \mathrm{~cm}$ oberhalb der tibiotalaren Gelenkebene gemessen 


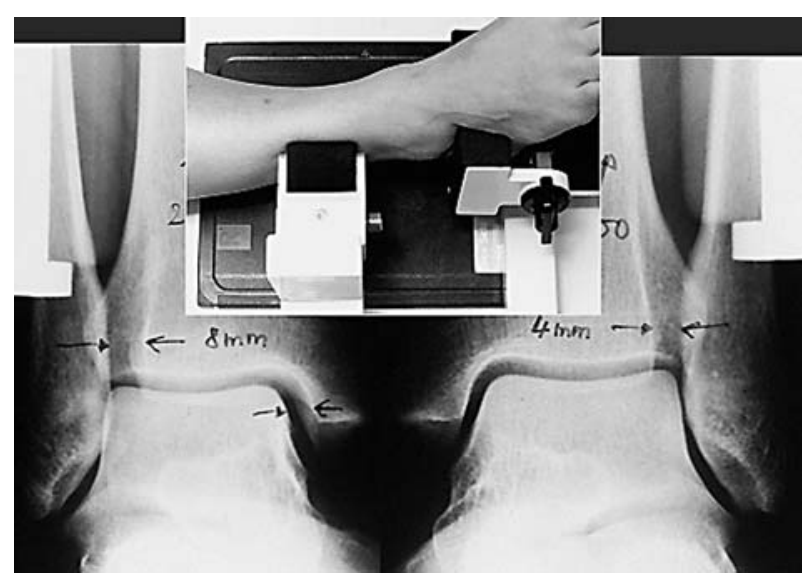

Abb. $2 \Delta$ Syndesmosenstressaufnahmen beidseits unter Standardbedingungen mittels eines neuen Zusatzteils für das Telos-Haltegerät. Der Rückfuß ist unterhalb des Talus fixiert. Die in der Mitte des Bilds erkennbare schwarze Rolle drückt ventral der Fibula die Tibia mit einem Druck bis zu $147 \mathrm{~N}$ nach medial, um eine mögliche Insuffizienz des distalen Syndesmosenkomplexes nachzuweisen. Dies ist im vorliegenden Fall im Seitenvergleich gut erkennbar

\section{Diagnostik}

Zur Evaluation von Fehlverheilungen nach OSG- oder Pilon-tibiale-Frakturen empfehlen sich grundsätzlich Belastungsaufnahmen im Stehen, wobei das obere Sprunggelenk a.-p. im Stehen mit $20^{\circ}$ Innenrotation aufgenommen wird, um eine präzise Darstellung des Gelenks als so genannten „mortise view“ zu haben. Mit dieser Aufnahme können nicht nur Hinweise für eine Fibulaverkürzung und Verdachtsmomente für eine Fehlrotation, sondern insbesondere messbare Kriterien, wie der so genannte Espace claire, die tibio-fibulare Überlappung und der mediale Gelenkspalt, exakt ausgemessen werden (Abb. 1). Nach eigener Beobachtung darf dabei der Espace claire nicht größer als $5 \mathrm{~mm}$ sein. Das tibiofibulare Überlappen sollte hingegen mehr als $5 \mathrm{~mm}$ betragen und der tibiotalare Spalt nicht mehr als 2-3 mm breit sein. Eine Verkürzung der Fibula kann prima vista durch die höher stehende so genannte Weber-Nase oder die Inkongruenz des so genannten Weber-Balls [8] erkannt werden, welche als diagnostische und auch als intraoperative Kriterien (Fallbeispiel 2) betrachtet werden. Die seitliche Belastungsaufnahme des Fußes mit OSG im Stehen zeigt, inwieweit das Rotationszentrum des Talus in Bezug zur Tibiaachse zu weit dorsal oder ventral steht. Sie lässt Fehlverheilungen der Tibiavorderkante (Fallbeispiel 3) oder des so genannten Volkmann-Dreiecks gut erkennen, zusätzlich eine potenziell zu weit dorsal oder ventral stehende Fibula.

Die präzisesten Informationen zur Lokalisation und zum Ausmaß der Fehlverheilung werden durch das koronare CT mit sagittaler Rekonstruktion erhalten (Fallbeispiel 3). Während eine isolierte chronische Instabilität des distalen Syndesmosenkomplexes klinisch durch die federnde Fibula feststellbar ist, kann sie durch handgehaltene Aufnahmen unter Durchleuchtung dokumentiert und mit einem neuen Zusatz zum TelosGerät unter Standardbedingungen quantifiziert werden (Abb. 2).

\section{Indikationen zur Operation}

Während bei supramalleolaren Fehlstellungen mit einem Varus $>5^{\circ}$, einem Valgus $>10^{\circ}$, einer Ante- oder Rekurvationsfehlstellung von $>15-20^{\circ}$ sowie einer Rotationsfehlstellung von $>10-20^{\circ}$ eine Korrektur [9] empfohlen wird, sind nach eigener Erfahrung Fehlstellungen im malleolaren Bereich, d. h. bei weitestgehendem direktem Gelenkkontakt, nur bis zu einer Fehlstellung jedweder Ebene von nur $5^{\circ}$ tolerierbar. Messbare Verkürzungen der Fibula, die eine Subluxation des Talus nach lateral erkennbar begünstigen, erscheinen ebenfalls korrekturbedürftig, desgleichen auch eine Fehlposition der Fibula in der Inzisur (CT-kontrolliert) von mehr als 2,5 mm abweichend zur gesunden Seite.

Da supramalleolare Pseudarthrosen oder hypertrophe, atrophe oder Infekt/ Defekt-Pseudarthrosen des Außenknöchels in der Regel Schmerzen bereiten, ist die Indikation zur Korrekturoperation dadurch grundsätzlich gegeben. Bei Innenknöchelpseudarthrosen, die oft
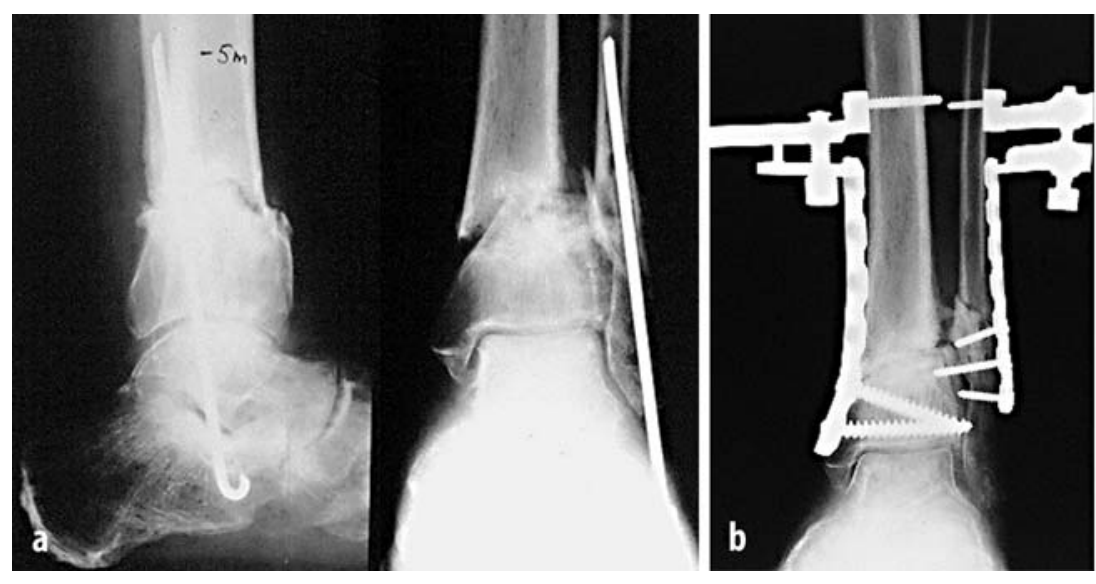

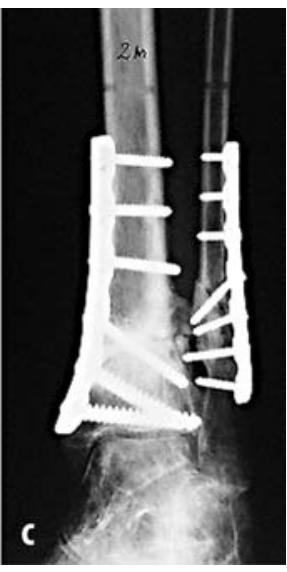

Abb. 3a-c $<$ Fall 1, supramalleolare Pseudarthrose (s. Text) 


\section{Unterschenkel- und obere Sprunggelenksfrakturen}
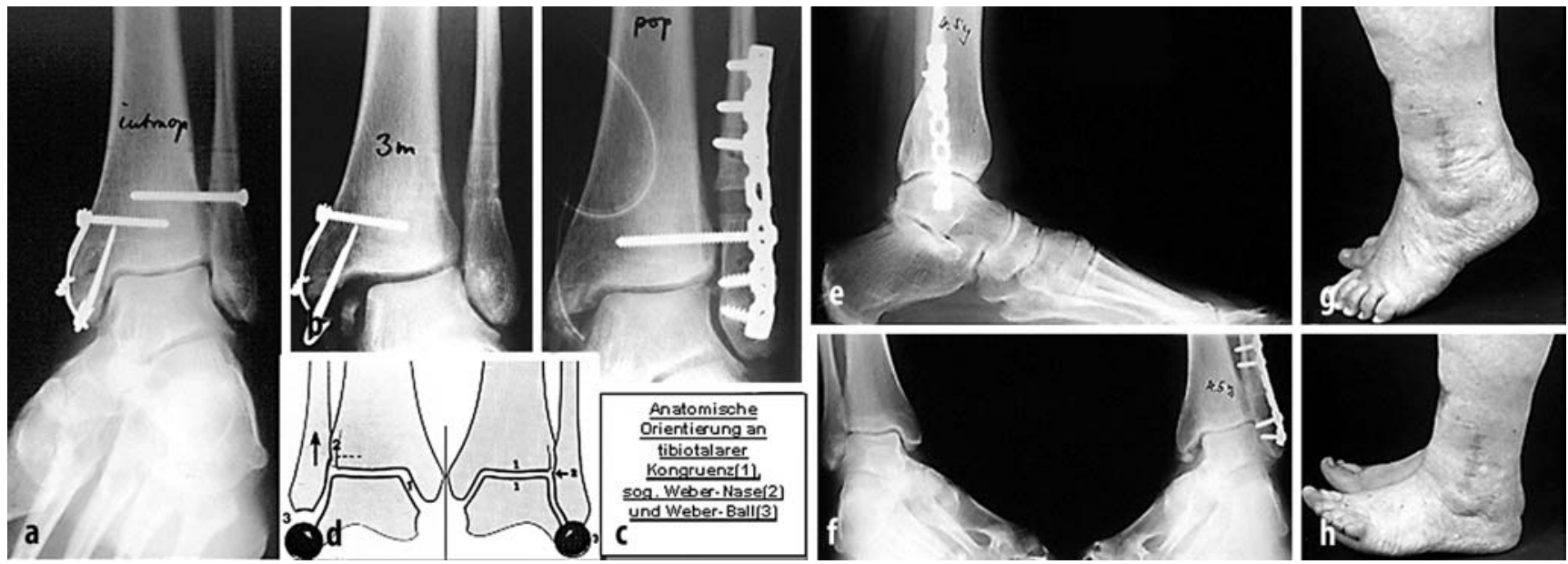

Abb. 4a-h $\Delta$ Fall 2, unter Verkürzung fehlverheilte Maisonneuve-Fraktur (s. Text)

schmerzlos sind, ist sie nur bei gegebener Instabilität oder einsetzenden Schmerzen zu stellen.

\section{Operative Therapie}

Das Ziel aller rekonstruktiven Maßnahmen bei Fehlheilung einer OSGoder Pilon-tibiale-Fraktur besteht in der Wiederherstellung eines anatomisch kongruenten, achsengerechten und ligamentär stabilen Gelenks, um hierdurch möglichst lange eine schmerzfreie Funktion zu erhalten. Voraussetzung hierfür sind die Beseitigung von Pseudarthrosen, die Korrektur der Achsen supramalleolar und malleolar, die Wiederherstellung der korrekten Längen- und Lagebeziehungen der Fibula zur Tibia sowie die Stabilität des distalen Syndesmosenkomplexes. Letztere ist ggf. durch eine modifizierte Castaigne-Ersatzplastik mittels halbem Peronaeus-longus-
Span zur anatomischen Wiederherstellung des vorderen und hinteren Syndesmosenbands sowie des biomechanisch sehr wichtigen Lig. tibio-fibulare interosseum zu erzielen [3].

\section{Fallbeispiele}

\section{Fall 1}

Bei einem 54-jährigen Patienten bestand 5 Monate nach distaler Unterschenkelfraktur mit Rush-Pin-Versorgung der Fibula und Oberschenkelgipsimmobilisation für 8 Wochen bei gleichzeitiger Marcurmarisierung wegen früherer Thrombembolien eine hypotrophe Pseudarthrose der supramalleolaren Tibia mit nicht sicherer Durchbauung der Fibula bei noch liegendem Rush-Pin (Abb. 3a).

Nach Entfernung des Rush-Pins erfolgte die Achsenkorrektur der Tibia mit Setzen einer 6 Loch breiten LCDCP an der medialen Tibia sowie einer kleinen 7-Loch-LCDCP an der Fibula, wobei die Pseudarthrose mit dem Plattenspanngerät beidseits maximal unter Druck gesetzt wurde (Abb. 3 b), sodass sie bereits nach 2 Monaten verheilte (Abb. $3 \mathrm{C}$ ).

\section{Fall 2}

Ein nicht allzu seltenes Phänomen stellt die Fibulafehlverheilung unter Verkürzung und Gabelinsuffizienz nach Maisonneuve-Fraktur dar. Bei einer 44-jährigen psychotischen Patientin nach Fahrradsturz mit folgender MaisonneuveFraktur und fraglich frischer Innenknöchelfraktur wurde der Innenknöchel mit einer Zuggurtung versorgt (Abb. 4a). Die Fibula hingegen erschien geringfügig verkürzt und wurde nicht mit 2, sondern nur mit einer 3,5er Kortikalisstellschraube transfixiert. 3 Monate nach der Erstversorgung und der zwischenzeitli-

Abb. 5a-e $\nabla$ Fall 3, fehlverheilte Pilon-tibiale-Fraktur (s. Text)
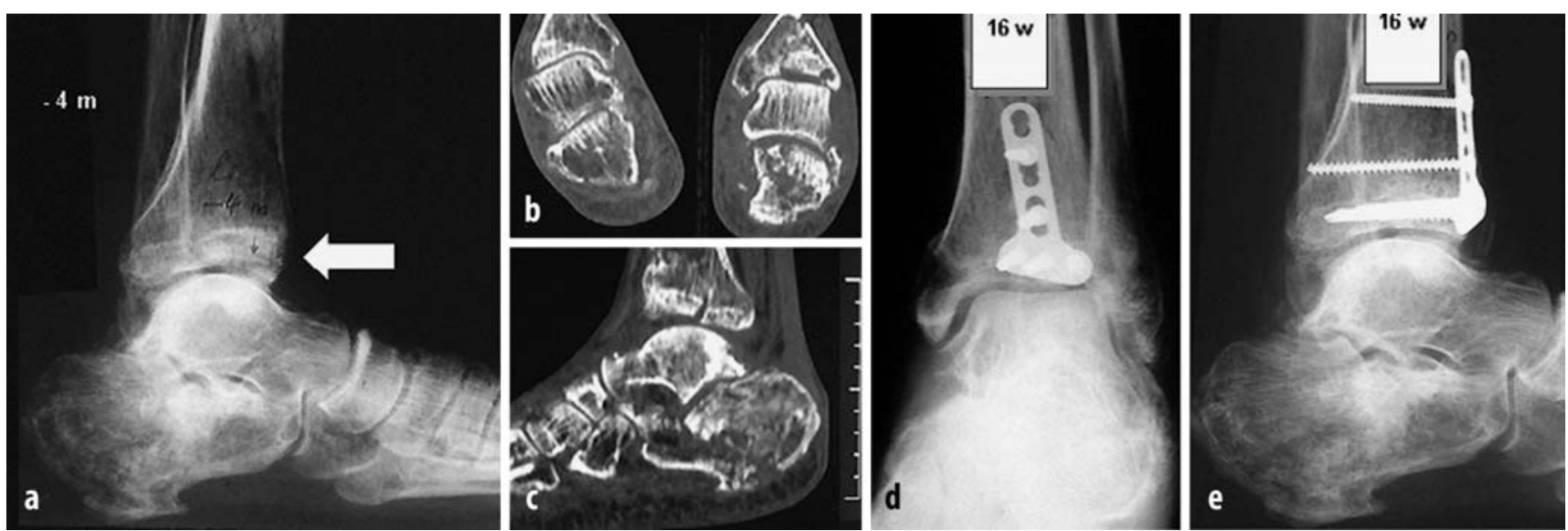
chen Stellschraubenentfernung (Abb. 4b) bestand eine massive Gabelinstabilität mit Verkürzung der Fibula, schwerer Subluxation des Talus nach lateral um nahezu $10 \mathrm{~mm}$ sowie eine Lockerung der Zuggurtung am Malleolus medialis. Nach Entfernung der Zuggurtung medial einschließlich medialer Gelenkrevision mit Entfernung des intraartikulären Narbengewebes ließ sich der Talus nach medial reponieren und die Fibula nach Osteotomie kongruent zum Gelenk unter $8 \mathrm{~mm}$ Verlängerung mittels Einsetzen eines kortikospongiösen Spans und Anlegen einer 7-Loch-Rekonstruktionsplatte einpassen (Abb. 4c), wobei zur sicheren distalen Syndesmosenheilung für 8 Wochen eine Stellschraube gesetzt wurde. Die intraoperative Orientierung zur korrekten Fibulalänge erfolgte über die Kontrolle der so genannten Weber-Nase (Abb. 4d) oder des gedachten Weber-Balls. 4 1/2 Jahre nach dieser Maßnahme ist die Patientin nach wie vor beschwerdefrei, hat eine unbegrenzte Gehstrecke bei radiologisch nur diskreter Arthrose (Abb. 4e,f) und guter Funktion (Abb. 4g,h).

\section{Fall 3}

Ein 44-jähriger männlicher Patient hatte vor 4 Monaten eine schwerste Polytraumatisierung erlitten, $u$. a. eine Pilontibiale-Fraktur (C1) sowie eine Kalka- neusfraktur (B2) rechts, die aufgrund der Polytraumatisierung auswärtig konservativ behandelt wurden mit jetzt bestehender Inkongruenz nicht nur im Subtalargelenk, sondern v. a. im Tibiotalargelenk (Abb. 5a). Die besonders im CT besser sichtbare Gelenkstufe (Abb. 5b,c) entspricht der Impaktion des Tuberculede-Chaput-Fragments. Zur Wiederherstellung des oberen Sprunggelenks wurden trotz massiver Inaktivitätsosteoporose 4 Monate nach dem Trauma die Osteotomie mit autologer Spanunterfütterung vorgenommen und die wiederhergestellte Gelenkkongruenz mit einem kleinen T-Plättchen gesichert. 16 Wochen nach diesem rekonstruktiven Eingriff war eine kongruente Verheilung erkennbar (Abb. 5d,e). Die sicher notwendige reorientierende subtalare Arthrodese nach fehlverheilter Kalkaneusfraktur sollte erst nach Rekalzifizierung und beim Auftreten von Schmerzen unter Vollbelastung, frühestens aber 1 Jahr nach dem Trauma, erfolgen.

\section{Literatur}

1. Bauer M, Jonsson K, Nilsson B (1985) Thirtyyear follow up of ankle fractures. Acta Orthop Scand 56: 103

2. Beck E (1978) Pseudarthrose des oberen Sprunggelenkes. Unfallheilkunde 133: 64

3. Grass R, Herzmann K, Biewener A, Zwipp H (2000) Verletzungen der unteren tibiofibularen Syndesmose. Unfallchirurg 103: 520-532

4. Hughes JL (1976) Corrective osteotomies of the fibula after defectively healed ankle fractures. J Bone Joint Surg Am 58: 728

5. Offierski CM, Hall JH, Harris WR et al. (1981) Late revision of fibular malunion in ankle fractures.J Bone Joint Surg Br 63: 460

6. Riess J (1955) Die Indikationsstellung zur operativen Behandlung frischer Brüche des inneren Knöchels. Chirurg 26: 103

7. Rosen H (1991) Reconstructive procedures about the ankle joint. In: Jahss MH (ed) Disorders of the foot and ankle, 2nd edn, vol III. Saunders, Philadelphia, p 2593

8. Weber BG (1981) Lengthening osteotomy of the fibula to correct a widened mortice of the ankle after fracture. Int Orthop 4: 289

9. Weise K, Weller S (1997) Supramalleoläre Korrekturosteotomien. In: Strecker W, Keppler P, Kinzl L (Hrsg) Posttraumatische Beindeformitäten: Analyse und Korrektur.Springer, Berlin Heidelberg New York, S 215

10. Weller S, Knapp U, Eck T (1977) Ergebnisse nach Korrektureingriffen am oberen Sprunggelenk. Unfallheilkunde 80: 213

11. Willenegger $H$ (1961) Die Behandlung der Luxationsfrakturen des oberen Sprunggelenkes nach biomechanischen Gesichtspunkten. Helv Chir Acta 28: 225

12. Zwipp H, Franck WM, Tscherne H (1996) Pseudarthrosen im Bereich des oberen Sprunggelenkes. Orthopäde 25: 463-469 